\title{
Opposing Actions of Androgen and Estrogen on In Vitro Firing Frequency of Neuronal Oscillators in the Electromotor System
}

\author{
Joseph E. Schaefer and Harold H. Zakon \\ Department of Zoology and Center for Developmental Biology, The University of Texas at Austin, Austin, Texas 78712
}

The South American knifefish (Apteronotus leptorhynchus), or brown ghost, produces a high-frequency $(600-1000 \mathrm{~Hz})$ sinusoidal electric organ discharge (EOD) with males discharging at higher frequencies than females. In addition, each fish has a unique EOD frequency within the frequency range of its gender. The electromotor circuit responsible for EOD production consists of a medullary pacemaker nucleus (PMN) and spinal electromotor neurons (EMNs).

In vitro spinal slice recording showed that, similar to the PMN, EMNs fire spontaneously at rates near the EOD frequency of each fish. The persistence of firing 2 weeks after high spinal transection demonstrated that spontaneous firing rate was intrinsic to the EMNs and was not dependent on presyn- aptic input. We confirmed that 11-ketotestosterone (11 kT) raised and $17-\beta$-estradiol (E2) lowered the EOD frequency of intact fish. Because electromotor cells fire spontaneously near EOD frequency, we investigated whether these steroids affect endogenous firing rates. Steroid implants were made in normal or spinally transected fish. Two weeks later, PMNs of normal fish and EMNs of transected fish were recorded in vitro. $11 \mathrm{kT}$ increased and E2 decreased the intrinsic firing rate of neurons in the PMN and the EMNs. Hormones shifted the intrinsic firing rates of EMNs, although they were synaptically isolated during the hormone exposure.

Key words: neuronal oscillators; steroids; electric fish; pacemaker; motoneuron; plasticity
The brown ghost, Apteronotus leptorhynchus, is a weakly electric fish found in tropical rivers of South America. It produces a quasisinusoidal electric organ discharge (EOD) that functions in electrolocation and social communication (Bullock and Heiligenberg, 1986). In this species, EOD frequency ranges from 600 to $1000 \mathrm{~Hz}$, with males discharging at higher frequencies than females (Meyer et al., 1987). In addition to this sexual dimorphism, there are stable individual differences in discharge frequency (Hopkins, 1972) in which EOD frequency fluctuates $<0.2 \%$ over the course of days to weeks (Bullock, 1969). As with many sexually dimorphic behaviors, EOD frequency is sensitive to steroid hormones: estrogen and testosterone lower basal EOD frequencies in immature and adult fish of either sex (Meyer et al., 1987; Dulka and Maler, 1994).

EOD frequency is set by neurons in a midline medullary nucleus called the pacemaker nucleus (PMN). This nucleus possesses two neuronal cell types (pacemaker and relay cells) that oscillate endogenously (Maler et al., 1983; Meyer, 1984; Dye and Heiligenberg, 1987). The activity of these cells initiates the EOD. Pacemaker cells make electrotonic connections with each other and with relay cells (Bennett et al., 1967). In vitro recordings of the PMN reveal that both pacemaker and relay cells isolated from their afferent input fire spontaneously at rates near the EOD frequency of each fish (Meyer, 1984; Dye and Heiligenberg, 1987). The relay cells project their axons down the spinal cord to form

\footnotetext{
Received Oct. 10, 1995; revised Jan. 24, 1996; accepted Jan 26, 1996.

This work was funded by National Institutes of Health Grant R01 NS25513 (H.Z.), and predoctoral support was provided by a National Institutes of Health developmental biology training Grant 5 T32 HD07296-05 (J.S.). We thank Kenneth Baimbridge for the generous gift of his R8701 calbindin antibody, Susan Gustavson for fish care, Gwen Gage and Janet Young for artwork, and Ying Lu for assistance in histology. We also thank the members of the Zakon and Pollak laboratories for their critical comments on this manuscript.

Correspondence should be addressed to Dr. Harold H. Zakon, Department of Zoology and Center for Developmental Biology, The University of Texas at Austin, Austin, TX 78712.

Copyright $\odot 1996$ Society for Neuroscience $0270-6474 / 96 / 162860-09 \$ 05.00 / 0$
}

electrotonic synapses with spinal electromotor neurons (EMNs), the axons of which comprise the adult electric organ (Bennett et al., 1971; Waxman et al., 1972; Kirshbaum, 1983). The summed activity of the EMN axons produces the EOD.

Unlike other weakly electric fish, the A. leptorhynchus electric organ continues to discharge for $\sim 24 \mathrm{hr}$ after the spinal cord is transected (severing the relay axons) (Dye and Meyer, 1986) (J Schacfer, unpublished data). Dye and Mcycr (1986) found that the post-transection EOD frequency was near the pretransection EOD frequency, suggesting that spinal neurons in the electromotor circuit also fire spontaneously (like pacemaker cells) at an intrinsic rate. Thus, in $A$. leptorhynchus, cells in both the PMN and the spinal cord fire spontaneously near each fish's own EOD frequency.

Because EOD frequency is shifted by steroids and neurons of the electromotor circuit are intrinsically "tuned" to oscillate at the EOD frequency, one or more neurons in the circuit are likely steroidsensitive. Compared with circuits underlying most vertebrate behaviors, the electromotor circuit is tractable with only three known cell types connected by electrotonic synapses. Within this circuit, one can compare homogeneous populations of cells among animals for variations in the membrane properties responsible for individual differences and hormone-induced changes in EOD frequency. This first requires the identification of possible steroid-sensitive cells in the circuit. In this study, we characterized the anatomy of the spinal electromotor regions to develop an in vitro preparation and locate the source of the spinal cord oscillations reported by Dye and Meyer (1986). We then examined the effects of androgen and estrogen on intact EOD frequency, and in vitro firing rates of both the PMN and the spinal cord oscillator.

\section{MATERIALS AND METHODS}

$A$. leptorhynchus were obtained commercially and kept in individual Plexiglas tanks that were part of a circulating 600 gallon system. The fish were in separate tanks for 2 weeks before use to allow their EOD 
frequencies to stabilize. Temperature and water conditions were kept stable during the experiment $\left( \pm 0.5^{\circ} \mathrm{C}\right)$.

\section{Anatomical methods}

Microscopy and image digitization. All slides were imaged under fluorescence and captured with a Cohu High Performance charge-coupled device camera and a Colorado Video 440A frame grabber. National Institutes of Health Image 1.4 was used on a Macintosh Quadra 800 to store and print the images.

Rhodamine-conjugated dextrans. To characterize the EMNs histologically, rhodamine-conjugated dextran crystals were used to back-fill the EMN somata. Fish were anesthetized with $0.075 \%$ 2-phenoxyethanol, and a small incision was made over the electric organ in caudal regions of the tail. A scalpel was used to cut the EMN axons in the electric organ under the incision, and dextran crystals were placed in contact with cut axons for 2 min before returning the fish to its tank. The dextrans were allowed to retrogradely label the EMNs for $7 \mathrm{~d}$. The tail was then removed and cut longitudinally in frozen $30 \mu \mathrm{m}$ sections. The sections were visualized with ultraviolet light and a rhodamine filter.

\section{Immunohistology}

Spinal cord tissue was processed for immunohistology by first fixing overnight in $4 \%$ paraformaldchyde in $0.1 \mathrm{M}$ PBS, pI 7.4 , and cryoprotecting for $1 \mathrm{hr}$ in $20 \%$ sucrose. Sections were flash-frozen and cut with a Bright freezing microtome (Hacker Instruments, Fairfield, $\mathrm{NJ}$ ) at $30 \mu \mathrm{m}$. Tissue sections were allowed to dry on slides and were surrounded with rubber cement and blocked for $1 \mathrm{hr}$ with $0.2 \%$ bovine serum albumin (BSA) in $0.1 \mathrm{~m}$ PBS, pH 7.4, with $0.5 \%$ Triton X-100 to permeabilize the cell membrane. The primary antibody $\left(1^{\circ} \mathrm{Ab}\right)$ was left on the sections for $24 \mathrm{hr}$ and rinsed with three washes (5 min each) of PBS/BSA-Triton. The secondary antibody $\left(2^{\circ} \mathrm{Ab}\right)$ was left on the slide for $1 \mathrm{hr}$ and rinsed with three washes ( 5 min each) of PBS/BSA in $0.5 \%$ Triton.

To label all axons in a section, we used $3 \mathrm{~A} 10$, which recognizes a neurofilament-associated protein and was purchased from the Developmental Hybridoma Bank (University of Iowa, Ames, IA). It is a monoclonal supernatant and was diluted $1: 1$ with BSA/PBS with $0.5 \%$ Triton $\mathrm{X}-100$. The antibody against calbindin- $\mathrm{D}_{28 \mathrm{k}}$ (R8701), which selectively labels pacemaker cells, relay cells (Maler, 1984), and EMNs, was generously provided by Dr. Kenneth Baimbridge (University of British Columbia, Vancouver, BC, Canada) and used at a dilution of 1:150 with PBS/BSA in $0.5 \%$ Triton. The appropriate $2^{\circ} \mathrm{Ab}$ for $3 \mathrm{~A} 10$ and R 8701 was used at a dilution of $1: 200$ with PBS/BSA in $0.5 \%$ Triton.

\section{Physiological methods}

EOD recordings. EOD was measured using two bare wires mounted on a Plexiglas rod. These two inputs were fed into a Grass P15 amplifier in a differential amplifying configuration. The P15 output was analyzed with a FLUKE voltmeter (Everett, WA) in the frequency-counter mode. EOD values were adjusted to an experimental standard temperature using a temperature correction factor $\left(Q_{10}\right)$ of 1.5 (Enger and Szabo, 1968).

\section{Spinal slice recordings.}

Tissue slices were superfused in a tissue slice chamber (designed by $\mathrm{R}$. Turner and L. Maler) with oxygenated $\left(95 \% \mathrm{O}_{2} / 5 \% \mathrm{CO}_{2}\right)$ artificial CSF (ACSF; $124 \mathrm{~mm} \mathrm{NaCl}, 2 \mathrm{~mm} \mathrm{KCl}, 1.25 \mathrm{~mm} \mathrm{KH}_{2} \mathrm{PO}_{4}$, $1.1 \mathrm{~mm} \mathrm{MgSO}_{4}-7$ $\mathrm{mM} \mathrm{H}_{2} 0,1.1 \mathrm{~mm} \mathrm{CaCl}_{2}-2 \mathrm{~mm} \mathrm{H}_{2} \mathrm{O}, 16 \mathrm{~mm} \mathrm{NaHCO}_{3}$, and $10 \mathrm{~mm}$ glucose) heated to the temperature of the animal's home tank $\left(26.0-28.0^{\circ} \mathrm{C}\right)$. This temperature was maintained by running the ACSF line through a bath heated with a World Precision Instruments (Gaithersburg, MD) Temperature Control Unit (accurate to $\pm 0.1^{\circ} \mathrm{C}$ ). The slices were visualized with a dissecting microscope and illuminated from above and below with fiber optic cables.

Fish were first lightly anesthetized in $0.075 \% 2$-phenoxyethanol. Then the caudal tip of the tail $(2 \mathrm{~cm})$ was removed and cut into $5 \mathrm{~mm}$ sections, and each section was mounted on a vibratome chuck using Vetbond $(3 \mathrm{M})$. The tail pieces were sectioned longitudinally in chilled $\left(4^{\circ} \mathrm{C}\right) \mathrm{ACSF}$ at a thickness of $150-250 \mu \mathrm{m}$.

Intra- and extracellular recordings were made with a microelectrode (20-50 M 2 ) placed "blindly" into the tissue because it was not possible to visualize the EMN somata. Action potentials were amplified with a World Precision Instruments INTRA 767 amplifier and stored on an analog Vetter 4-channel VCR data recorder with a sampling rate of 22 $\mathrm{kHz}$. Spike frequency was analyzed off-line by transferring the data via a
World Precision Instruments Universal Signal Manifold and Data Translation A/D board to a Standard 286 AT clone and then analyzed with the computer program SPIKE at a sampling rate of $20 \mathrm{kHz}$. This software was used to calculate average firing frequencies during $25 \mathrm{msec}$ windows sampled every $10 \mathrm{sec}$ during a continuous playback from the Vetter recorder.

Two firing patterns were observed and analyzed separately. Cells that fired continuously at a stable frequency $( \pm 5 \%)$ were called constant cells, and their frequency was averaged over $1 \mathrm{~min}$. Cells that were active in periodic bursts were called cycling cells. Average firing frequency for cycling cells was found by averaging the peak frequency achieved during each burst. To be considered for analysis, cells were required to exhibit a consistent behavior for $2 \mathrm{~min}$. The second minute was analyzed with SPIKE. A minimum of five cells per fish were required for the data to be included in the study. $\Lambda$ simple regression was performcd on the data, and the significance of the correlation was tested using the Pearson's $r$ correlation.

Pacemaker recordings. The PMN was obtained by anesthetizing the fish with 2-phenoxyethanol $(0.075 \%)$ and then removing the brain. The brain was placed into cold, oxygenated ACSF and dissected further to isolate the brainstem. The brainstem containing the PMN was placed ventralside-up into the tissue slice chamber and covered with saline throughout the experiment. Superfusion was halted during recording to keep the tissue from moving. This sometimes resulted in small changes in recording temperature $\left( \pm 0.5^{\circ} \mathrm{C}\right)$, and becaluse the $\mathrm{EOD}$ is a temperaturedependent process, all PMN firing frequencies were adjusted using a $Q_{10}$ of 1.5 (Enger and Szabo, 1968) to a common temperature for all experiments. Extracellular field potential recordings were obtained from sevcral different areas of the PMN and then averaged. If field potentials could not be detected, intracellular recordings of firing frequency from a minimum of five pacemaker and relay cells were averaged.

Spinal transection and steroid implant. Fish were anesthetized in 2-phenoxyethanol until respiration ceased. An incision was made along the dorsal midline $1-2 \mathrm{~cm}$ behind the head. An opening was made to the spinal cord through the vertebral arches, the cord was severed with forceps, and the incision was sutured closed. The entire procedure lasted $5 \mathrm{~min}$ in most cases. The fish were respired and checked for the absence of an EOD as a positive indication of transection. The transection was verified further immunohistologically in several of the fish. This was done by removing the tail piece for vibratome slicing, cutting it into several $0.5 \mathrm{~cm}$ sections, and placing one of them in $4 \%$ paraformaldehyde for later immunohistology. The other sections were sliced and used in the recording chamber.

Fish in the implant group received a SILASTIC (Dow Corning, Corning, NY) capsule $(0.5 \mathrm{~cm})$ filled with $1 \mathrm{mg}$ of the nonaromatizable androgen 11 ketotestosterone ( $11 \mathrm{kT}$ ), $1 \mathrm{mg}$ of 17 - $\beta$-estradiol (E2; Sigma, St. Louis, MO), or an empty control capsule. The capsule was placed within the peritoneal cavity (Keller et al., 1986) and remained in the fish for the entire study, which was verified postmortem. This procedure results in steroid blood levels of a few to $10 \mathrm{ng} / \mathrm{ml}$ (Keller et al., 1986; Zakon et al., 1993).

The species EOD frequency range for $A$. leptorhynchtos is $600-1000 \mathrm{~Hz}$. The fish were divided into high $(>800 \mathrm{~Hz})$ and low $(<800 \mathrm{~Hz})$ EOD frequency groups. To maximize the effects of steroid implant, we implanted fish in the low-frequency group with a steroid to raise EOD frequency $(11 \mathrm{kT})$ and fish in the high-frequency group with a steroid to lower EOD frequency (E2). Control fish received empty capsules and were matched to their respective treatment groups by their mean EOD frequencies.

In the steroid implant experiments, the post-treatment EOD frequency and PMN and EMN firing rates were subtracted from the fish's preimplant EOD frequency to yield a difference frequency. Difference frequencies from all of the cells in a fish were averaged to provide one value per fish. The $11 \mathrm{kT}$ and E2 implant groups were compared with their corresponding control groups using a single-variable ANOVA with Scheffe's and Fischer's post hoc analyses at $95 \%$ significance.

Lucifer yellow dye injections. The recording elcctrode was back-filled with a $3 \%$ solution of Lucifer yellow dye. Then the rest of the electrode was filled with $0.1 \mathrm{M} \mathrm{LiCl}$. The dye was iontophoresed with a $2 \mathrm{nA}$ hyperpolarizing pulse at a $50 \%(500 \mathrm{msec})$ duty cycle. The dye was allowed to diffuse in the tissue for $30 \mathrm{~min}$ before fixation in $4 \%$ paraformaldehyde in $0.1 \mathrm{M}$ PBS, pH 7.2, overnight. The tissue was dehydrated in ethanol, cleared in methyl salicylate, whole-mounted on a depression slide, and viewed with fluorescence microscopy. 


\section{RESULTS}

\section{Anatomical and histological characterization of} electromotor circuit

It was necessary to characterize first the organization of the electromotor circuit to provide an anatomical context for the spinal cord in vitro preparation. EMNs, which are clustered dorsal and lateral to the central spinal canal, were conclusively identified by dextran back-fills from the electric organ. EMNs are $30-50 \mu \mathrm{m}$ in diameter, largely adendritic with a few thin dendrites extending from the somd, and have a single axon exiting through a ventral root (Fig. $1 A, B$ ). The EMN axons fasciculate and make up the electric organ in this species.

Because spinal transection was to be used later in this study to remove the presynaptic relay inputs to the EMNs, we wished to identify the relay axons in cross-section. All axons in a section were visualized with $3 \mathrm{~A} 10$, which recognizes neurofilaments (Fig. $1 C, E)$. Two prominent dorsolateral tracts of large-diameter $(10-20 \mu \mathrm{m})$ fibers were observed that correspond to previous descriptions of the spinal relay tracts (Bennett, 1971). We directly confirmed their identity by injecting Lucifer yellow after physiological characterization. This showed that single filled axons branch extensively as far as they could be visualized in whole mounts over several spinal segments (Fig. 2). These axons send collaterals to form a dense neuropil of fibers surrounding the EMNs. Anti-calbindin-D ${ }_{28 \mathrm{k}}$ (R8701) selectively labeled these relay axons, theit collaterals, and the EMNs (Fig. $1 D, F$ ).

\section{Spontaneous activity in vitro}

Spinal slice recordings were made from fish with EODs across the A. leptorhynchus frequency range $\left(600-1000 \mathrm{~Hz}\right.$ at $\left.\sim 27.0^{\circ} \mathrm{C}\right)$. This is the first investigation of the in vitro activity of the spinal electromotor system. It revealed that in the slice preparation these cells are spontaneously active in either of two distinct patterns. Both patterns could be found in any one slice of tissue, but in no case did a cell switch between the two behaviors during a recording. Cells that we termed "constant" (Fig. $3 A$ ) fluctuated in firing frequency by as little as $2 \%$ during a recording lasting sometimes up to $10 \mathrm{~min}$. The other type displayed periodic fluctuations of firing frequency, membrane potential, and spike amplitude; thus, we called thcm "cycling" cells (Fig. 3B). These cells reached their peak frequency within milliseconds of their quiescent phase accompanied by a $10-20 \mathrm{mV}$ depolarization of the membrane. Then firing frequency immediately slowed, spike amplitude increased, and the membrane potential hyperpolarized until the cell suddenly hyperpolarized 5-10 $\mathrm{mV}$ in one step and stopped firing.

The average firing frequency of constant cells and the average peak frequencies of cycling cells were plotted against the original intact EOD frequency (see Fig. $3 C$ ) to reveal a significant correlation ( $n=13, r=0.681, p<0.02$ ). Thus, cells within each fish's spinal cord intrinsically oscillate at rates near its EOD frequency.

In the caudal tip of the spinal cord, there are only two cells known to be involved in the generation of the EOD: relay axons and EMNs. Lucifer yellow iontophoresis was used to label the last cell recorded in many experiments. The results of these fills did not identify conclusively either cell type to be the single source of spinal oscillations or the cycling firing pattern. Of a total of 10 cells filled, 8 were made in cycling cells. Seven of the cycling cells were identified later as relay axons (Fig. 2). Although this suggests that cycling arises from relay axons, the remaining fill was in an EMN. The only two fills made in constant cells labeled one relay axon and one EMN (data not shown). These ambiguous results might be attributable to the difficulty of localizing the source of activity in an electrically coupled system. To focus on the electrical behavior of the EMNs in isolation, we chose to eliminate the relay axons by transecting the spinal cord 2 weeks before in vitro recording.

\section{Tuned oscillation in EMNs}

Spinal transection made just caudal to the thoracic cavity 2 weeks before slice recording causes degeneration of relay axons. The success of this procedure was verified by the long-term absence of an EOD. Of the tail pieces sliced for in vitro recording, one was fixed and processed for $3 \mathrm{AlO}$ immunohistology to verify the absence of all relay axons and their collaterals after the experiment (Fig. 4A).

To examine the remaining spontaneous electromotor activity, the recording electrode was guided to the ventral third of the spinal slice, where the EMN axons from adjacent spinal segments converge to exit through the ventral root. Although relay axons were now absent from the slice preparation, spontaneous electrical activity was still detected. All cells recorded in this experiment fired in a constant pattern ( $n=95$ cells). Because relay axons were absent from the spinal cord, the remaining high-frequency activity could have originated only from EMNs, and Lucifer yellow injections confirmed this ( $n=5$ fish; Fig. $4 B$ ).

The mean firing rates of all sampled EMNs in a fish were averaged and plotted against each fish's EOD frequency measured before transection (Fig. 5). This revealed that EMNs oscillate at constant rates similar to each individual fish's EOD frequency despite being isolated from the PMN $(r=0.77, n=15$ fish, $p<0.001$ ).

\section{Effects of steroids on the electromotor circuit}

Fish were divided into groups to be used in three separate experiments. In these experiments, we looked at the effects of steroids on EOD frequency and the in vitro firing frequency of the PMN and EMNs. Equal numbers of fish were divided into high- and low-frequency groups (see Materials and Methods) for each experiment and given either an empty control capsule or one containing steroid. The experimental fish with a low EOD frequency $(<800 \mathrm{~Hz}$ ) were given $11 \mathrm{kT}$, and high EOD frequency fish $(>800$ $\mathrm{Hz}$ ) received $\mathrm{E} 2$.

Two to three weeks after implant, the effects of steroid treatment (or implant alone in the case of controls) were assessed (see Materials and Methods for data analysis) in three different experiments: PMN (3 PMNs/group), EMN (5 fish/group), and intact EOD frequency ( 5 fish/group). The results of the three experiments indicate that $11 \mathrm{kT}$ significantly increased EOD frequency, in vitro $\mathrm{PMN}$ firing rate, and in vitro EMN firing rate. In contrast, E2 decreased EOD frequency, in vitro PMN firing rate, and in vitro EMN firing rate.

\section{DISCUSSION}

\section{Anatomical organization of spinal electromotor circuits}

Immunocytochemistry, Lucifer yellow injection, and retrograde labeling with dextrans revealed two aspects of the spinal electromotor circuit (EMC) not previously reported. The first of these was the positive labeling of the EMNs with antibodies to calbindin- $\mathrm{D}_{28 \mathrm{k}}$ (R8701). Our results and those of Maler et al. (1984) indicate that all cells in the electromotor pathway are calbindin- $\mathrm{D}_{28 \mathrm{k}}$-positive. Second, Apteronotus EMNs have been reported to be adendritic in previous studies using plastic sections (Bennett, 1971; Waxman et al., 1972; Bennett et al., 1978; Anderson et al., 1984). However, we now show using a retrograde 

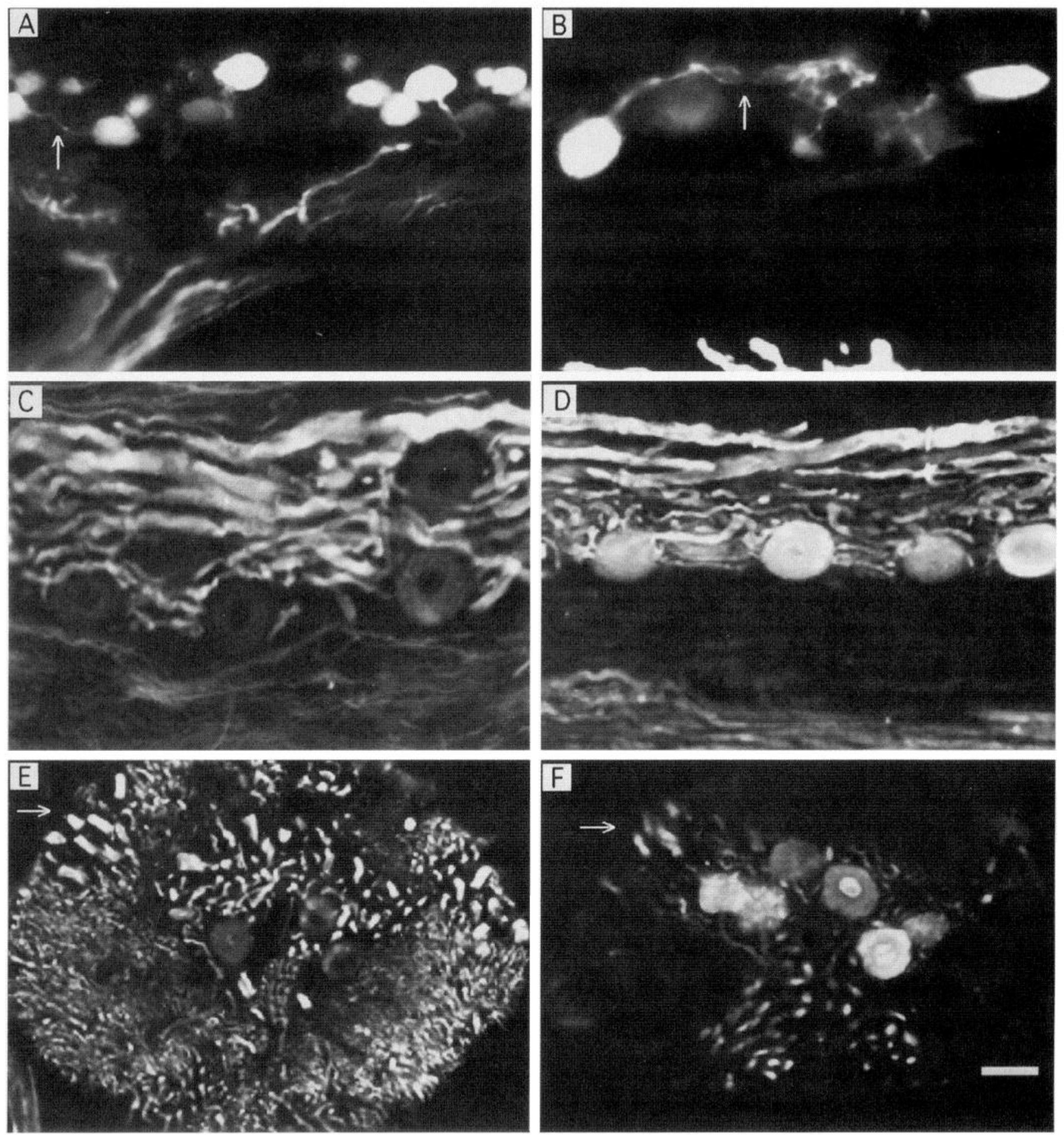

Figure 1. Spinal electromotor circuit anatomy. $A, B$, EMNs back-filled from the electric organ with rhodamine-conjugated dextrans. The sections are longitudinal with the ventral root at the bottom. Note the dendrites in $A$ and $B$ (arrow). $C$, Longitudinal section labeled with $3 \mathrm{~A} 10$ (which recognizes a neurofilament-associated protein). The central neuropil of relay axon collaterals surrounds the EMN somata, which do not label as strongly for neurofilaments. $D$, Longitudinal section labeled with $\mathrm{R} 8701$ (which recognizes calbindin- $\mathrm{D}_{28 \mathrm{k}}$ ) shows anatomy similar to that seen with $3 \mathrm{~A} 10$. $E$, Cross-section labeled with 3A10 shows relay axons (arrow) tracts in both dorsolateral quadrants. Their collaterals form the central neuropil and surround the EMN somata, which lie dorsal and lateral to the central spinal canal. $F$, Cross-section labeled with R8701. Only relay axons (arrow) and EMNs label with R8701. Scale bar, $50 \mu \mathrm{m}$.

tracing molecule that EMNs possess a small number of thin dendrites. The dendrites persist after spinal transection completely ablates the central neuropil of relay collaterals, eliminating the possibility of them being presynaptic in origin. Furthermore, the $M_{\mathrm{r}}$ of the dextrans ( $\left.10 \mathrm{kDa}\right)$ used in back-fills far exceeds the size limit for molecules to pass through gap junctions (1 kDa) (Bennett et al., 1991). What role the dendrites serve in the electromotor output is unclear at this point. 
Figure 2. Spinal slice whole mount after in vitro recording from and Lucifer yellow injection into a relay axon. The brightest label corresponds to the site of microelectrode penetration. A large number of collaterals can be seen to branch from the main axon. Scale bar, $100 \mu \mathrm{m}$.

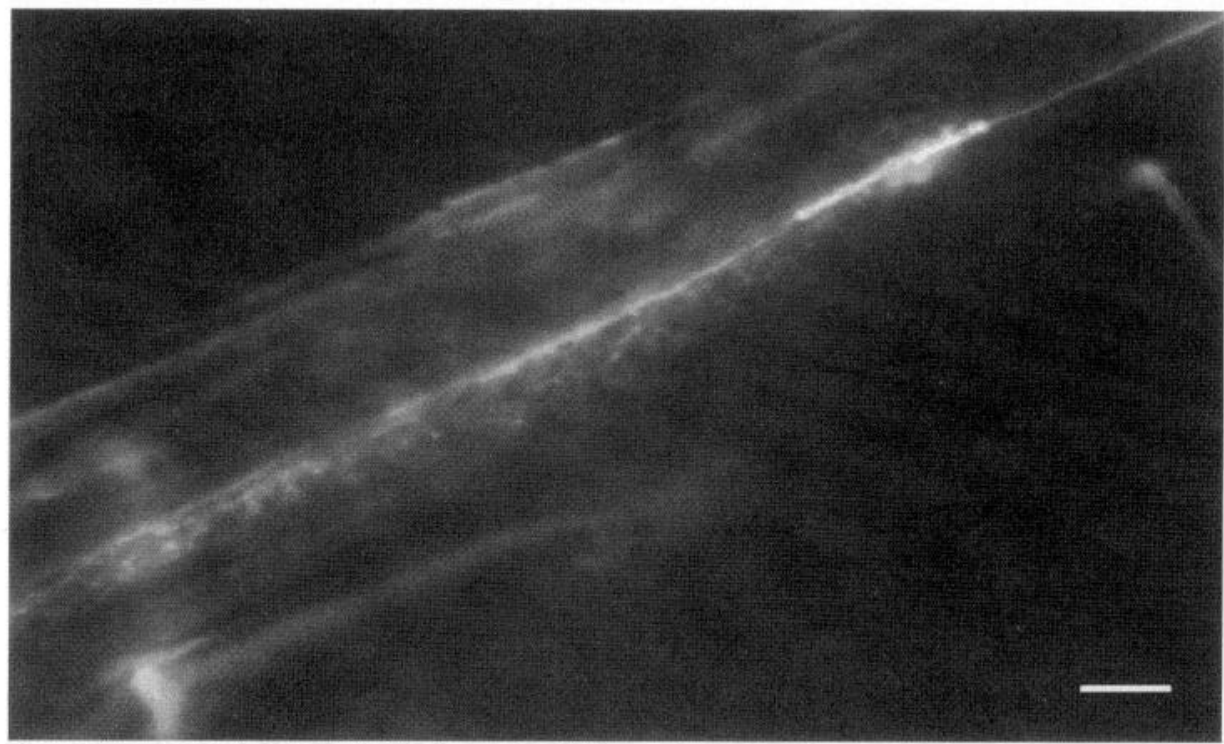

Finally, whole mounts of Lucifer yellow fills of relay axons show that they create a dense neuropil of fibers surrounding the EMNs, which has not been described previously (Bennett et al., 1978). Single filled relay axons extended numerous collaterals over the entire length that could be visualized in a whole-mount section (often exceeding one spinal segment). The highly branched relay axon morphology can also be seen in frozen sections labeled with $3 \mathrm{~A} 10$ or anti-calbindin- $\mathrm{D}_{28 \mathrm{k}}$. The numerous branches and EMN contacts made by each relay axon functionally couple large groups of EMNs. This certainly plays a critical role in synchronizing the activity of the EMN population required for EOD production.

\section{Electromotor neurons as intrinsic oscillators}

Electromotor cells in a slice preparation of spinal cord fire spontaneously at either a constant frequency or in bursts of activity separated by periods of quiescence. We originally hypothesized that the two firing patterns might correspond to the two cells types present in the slice (EMNs and relay cell axons), but intracellular Lucifer dye injection did not support this. EMNs and relay axons are coupled by gap junctions; therefore, a recording electrode in one cell type might pick up activity originating from the other. To focus on one cell type, a complete spinal transection was made 2 weeks before recording, eliminating relay axons from the preparation. Consequently, EMNs fired only in a constant pattern at rates correlated to intact pretransection EOD frequency. This suggests that the EMNs changed their firing pattern from cycling to constant after transection or that the presence of cut relay axons in the slice preparation originally produced the cycling firing pattern.

The simplest explanation given the data presented here and elsewhere is that cycling arises from the connection of EMNs to cut relay axons. Evidence for this hypothesis comes from the spinal transection experiment of Dye and Meyer (1986). If one monitors EOD frequency after transection, the entire electric organ is seen to exhibit cycling activity. This persists for several hours after transection and looks similar to what we describe here as the cycling firing pattern. The post-transection EOD is characterized by periods of high-frequency, high-amplitude output interspersed with periods of very low-amplitude background activity. (J. Dye, personal communication) (J. Schaefer, unpublished observations). After $24 \mathrm{hr}$, this background activity is all that remains of the EOD. Because our in vitro data show that EMNs are still oscillatory up to 1 month after transection, this lowamplitude background activity is probably EMNs now firing out of synchrony. The common condition between the in vitro EMN cycling and in vivo EOD cycling is the presence of cut relay axons coupled to EMNs. The disappearance of EOD cycling $24 \mathrm{hr}$ after transection corresponds to the known time course of relay collateral degeneration after spinal transection (Schaefer et al., 1994).

\section{Coupled oscillators}

The oscillatory EMNs found in A. leptorhynchus are thus unique within the order Gymnotiformes. In other species studied so far, pacemaker cells "drive" the otherwise silent EMNs. Evidence presented here and elsewhere (Dye and Meyer, 1986; Schaefer and Zakon, 1993) demonstrates that the PMN in A. leptorhynchus only entrains the already active EMNs. Depending on the species, Apteronotid EMNs are required to fire between 500 and $1800 \mathrm{~Hz}$ (Alves-Gomes et al., 1995). At these rates, it may be easier for the PMN to entrain or synchronize already active EMNs through the relay axon network than to depolarize them cycle-by-cycle.

The spontaneous oscillations of brown ghost EMNs place constraints on several well characterized reflexive and social modulations of EOD frequency. In Eigenmannia and Hypopomus, the jamming avoidance response and other social signals (chirps, decelerations, etc.) require transient increases or decreases in EOD frequency (Hagedorn and Heiligenberg, 1985; Kawasaki et al., 1988). However, Apteronotids only show EOD frequency increases and are unresponsive to stimuli that elicit frequency decreases in other species. It is easy to see how an increase in PMN firing frequency might drive EMNs to higher rates by depolarizing them earlier in their activity cycle. However, if the PMN slowed its firing rate, this may have no effect on the endogenously active EMNs, or it could allow each cell to fire at its intrinsic rate, thereby desynchronizing the population.

\section{Effects of steroids on the EMC neuronal firing rate}

In $A$. leptorhynchus, females normally discharge at significantly lower rates than males (Hagedorn and Heiligenberg, 1985; Meyer et al., 1987). Thus, it is not surprising that estrogen lowers and 11 kT raises EOD frequency (Meyer et al., 1987; Zakon et al., 1993) (M. Zucker and H. Zakon, unpublished data). The fish in our 
A

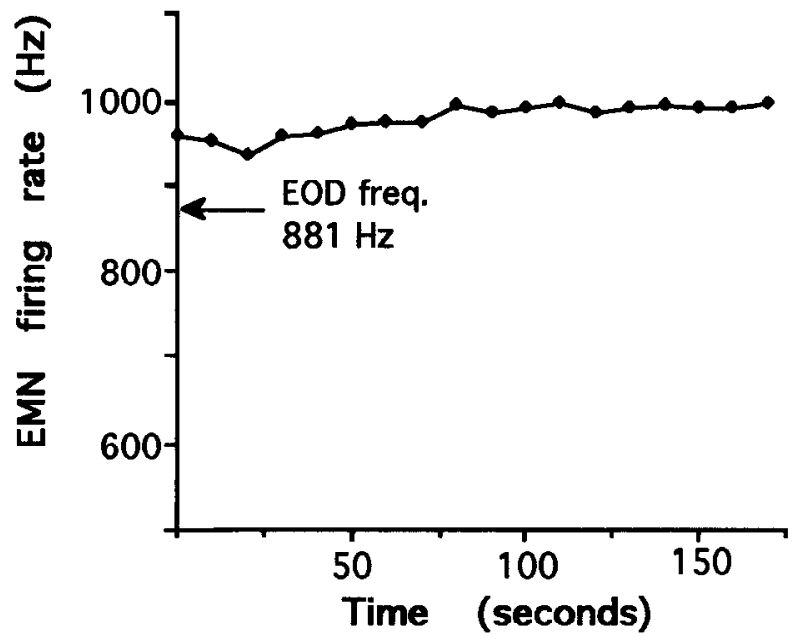

B

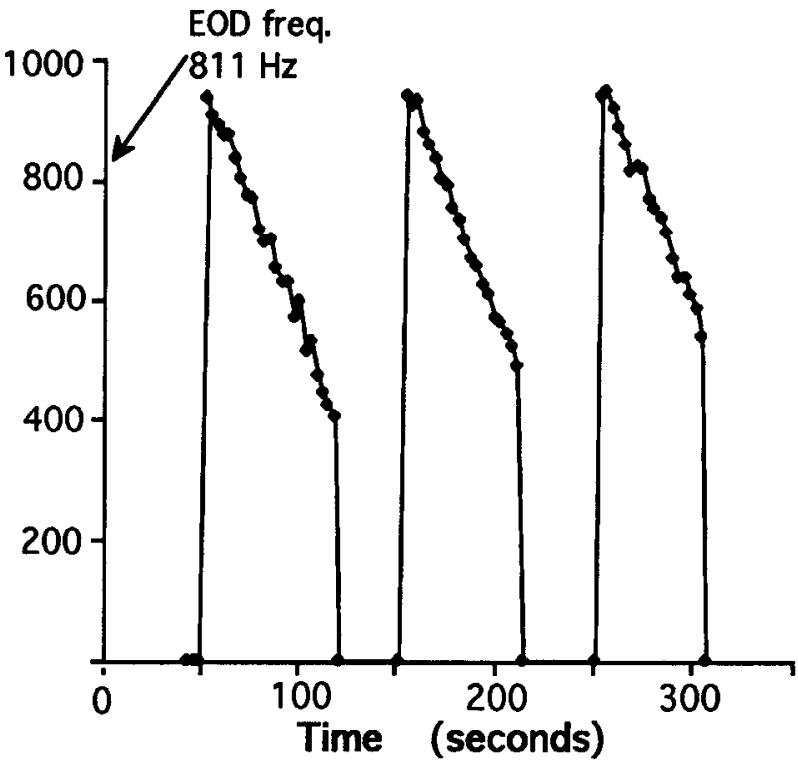

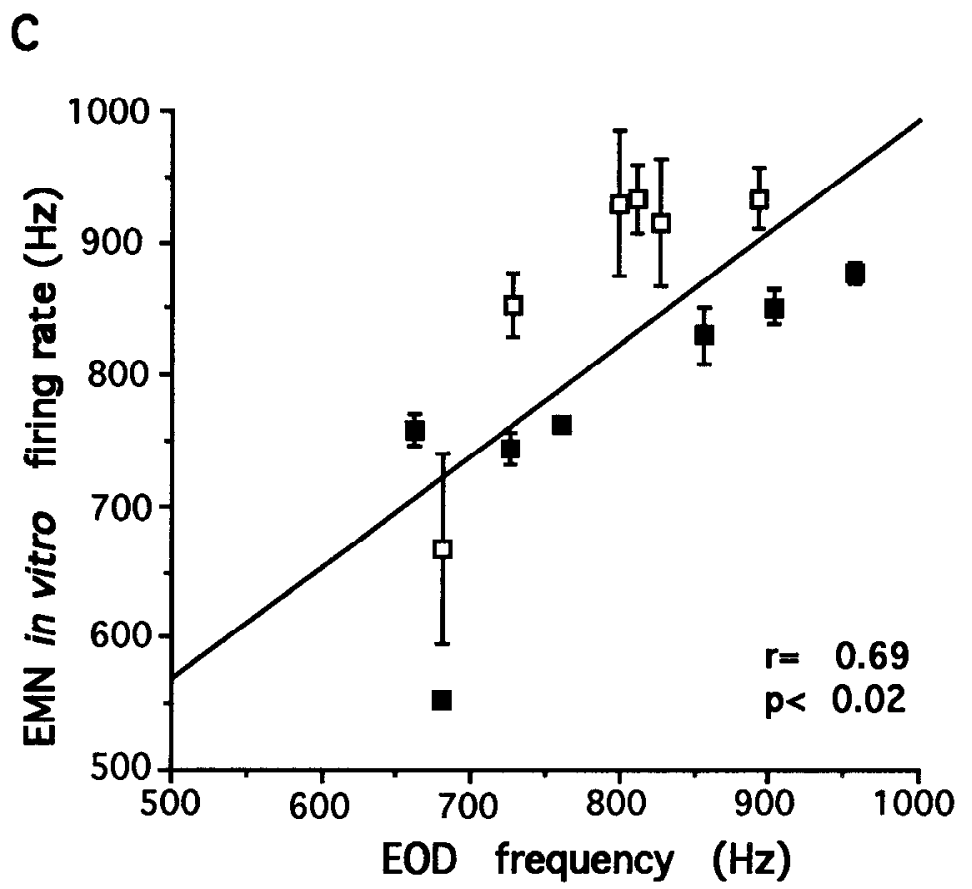

Figure 3. Analysis of in vitro activity in the spinal tissue slice. $A$, Firing frequency of a typical constant cell during a 3 min intracellular recording. $B$, Firing frequency of a typical cycling cell during a $4 \mathrm{~min}$ intracellular recording. $C$, Constant cell firing rate (filled squares) and the cycling cell average peak frequencies (open squares) were averaged for each fish and plotted against its original intact EOD frequency. Error bars represent the SEM.

study were neither sexed nor gonadectomized. Nevertheless, E2 lowers and $11 \mathrm{kT}$ raises EOD frequency in gonadectomized fish of both sexes (M. Zucker and H. Zakon, unpublished data).

Steroids could induce long-term FOD frequency changes by acting as follows: (1) directly on an EMC neuron to induce long-term changes in its intrinsic firing frequency; (2) on a presynaptic neuron that tonically influences the firing frequency of the electromotor circuit neuron, but that would allow that neuron to return to its original frequency if removed; or (3) on a presynaptic neuron that induces permanent changes in the spontaneous firing frequency of EMC neurons that will remain after removal of presynaptic inputs.

In vitro recordings consistently showed that PMNs from control fish in the high EOD frequency group fired at rates higher than their EOD frequency, whereas PMNs from fish in the lowfrequency group fired at rates lower than their EOD frequency. Although the source of this variation is unknown, on this effect is superimposed the frequency shift induced by steroid implant. A statistical comparison between the difference frequencies in steroid-implanted fish and their frequency-matched controls re- 

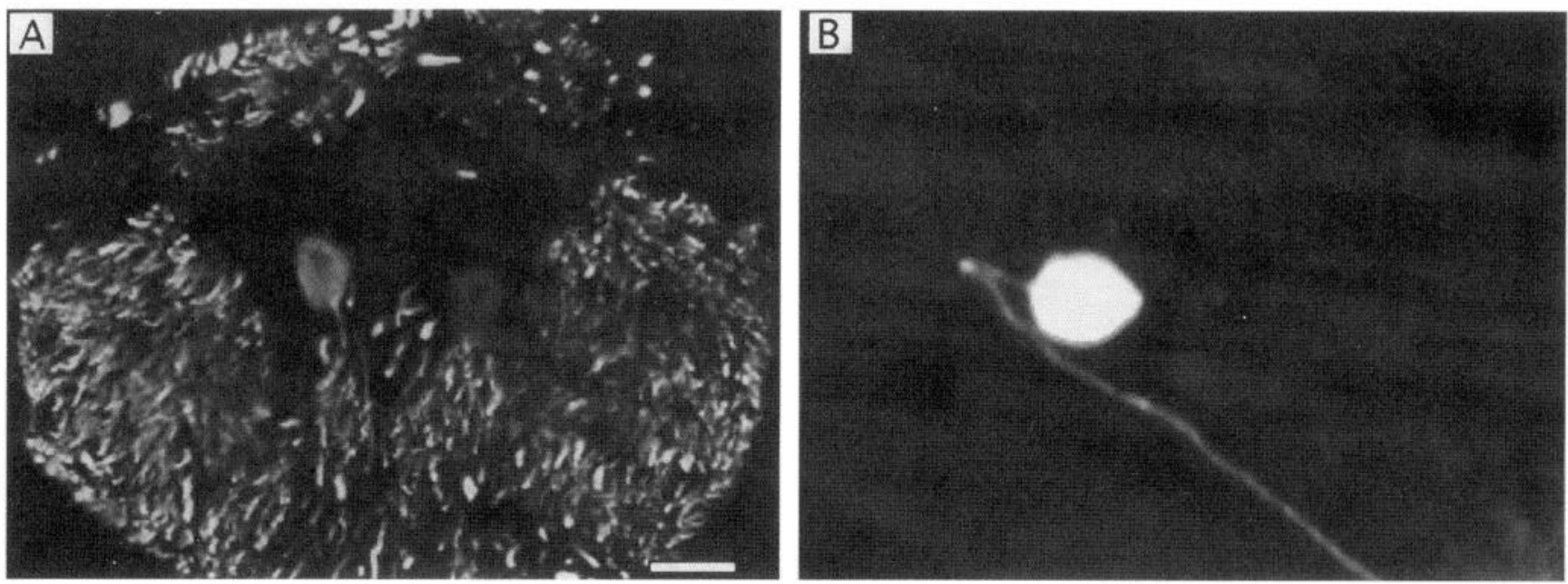

Figure 4. Histology of spinal cord from transected fish. A, Cross-section of caudal spinal cord $30 \mathrm{~d}$ after spinal transection, labeled with $3 \mathrm{~A} 10$. Note the absence of the relay axons and collaterals. $B$, Whole mount of a longitudinal spinal slice in which a cell received Lucifer yellow injection. The tissue was from a spinally transected fish. Scale bar, $50 \mu \mathrm{m}$.

vealed a steroid-sensitive component. This is supported further by the fact that E2 and $11 \mathrm{kT}$ produce changes in PMN firing frequency similar to what is seen in the EOD frequency and the in vitro EMN firing rate of implanted fish (Fig. 6).

The diencephalic prepacemaker nucleus and mesencephalic prepacemaker nucleus are the only known input to the PMN and are responsible for modulations of firing frequency that produce socially relevant EOD behaviors (Kawasaki et al., 1988). Because the PMN received synaptic input from these nuclei during the

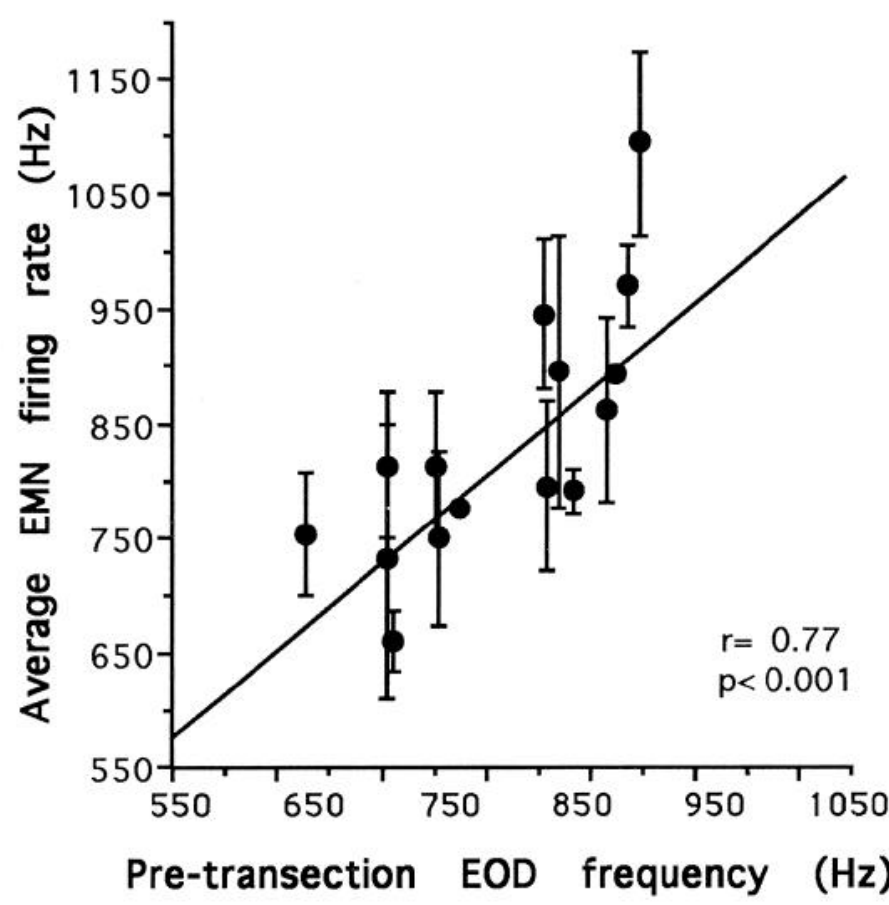

Figure 5. Analysis of spontaneous in vitro EMN activity in spinally transected fish. All in vitro recordings made in tissue from transected fish were of a constant firing pattern. The mean firing rates for all of the recorded cells of a fish were averaged and compared with the original pretransection EOD frequency. Error bars represent the SEM. hormone treatment, we cannot determine whether steroids acted directly on the nucleus or through these inputs. Long-term steroid-induced changes in spontaneous firing frequency also occurred in EMNs. However, these changes occurred in the absence of relay axon inputs. Thus, steroids do not act on EMNs through tonic input from the PMN. Instead, steroids might act directly on EMNs or other cells in the spinal cord.

These changes in the spontaneous firing rates of neurons in the EMC are likely mediated by steroid actions on ion currents of these cells. Previous studies on isolated Apteronotus PMN revealed that its firing frequency is extremely sensitive $(50-100 \mu \mathrm{M})$ to 4-aminopyridine (4-AP) (Dye, 1991). Sensitivity to 4-AP infers the presence of a rapidly activating, but transient, outward potassium current $\left(\mathrm{K}_{\mathrm{A}}\right) . \mathrm{K}_{\mathrm{A}}$ is often responsible for setting repetitive firing rates (Hille, 1992) and would be a likely target for steroid modulation.

Spontaneously firing neurons also require an endogenously active inward current. Thus, the firing rate of EMC neurons could also be influenced by steroidal modulation of an inward current. Candidates for such steroid-sensitive inward currents would include the $\mathrm{I}_{\mathrm{h}}$ cation channel, which activates with hyperpolarization (Hille, 1992), and persistent (leak) sodium currents (Gage et al., 1989). In the gymnotid Sternopygus, for example, $5 \alpha$-dihydrotestosterone lengthens the inactivation time constant of electrocyte voltage-activated sodium current (Ferrari et al., 1995).

EMC neurons respond to elevated levels of androgen and estrogen with opposite changes in firing frequency. To produce such an effect, the two hormones could act with opposite effects on the same ion current, or one could regulate an inward current while the other regulates an outward current. Determining the influence of steroids on the magnitude and kinetics of the various ion currents of EMC cells requires voltage-clamp analysis. In the PMN of Apteronotus, this is difficult because of extensive dendritic arborization and electrical coupling. However, EMNs are spherical, largely adendritic, and abundant in caudal regions of the spinal cord. Using the techniques of Turner et al. (1994), we have enzymatically dissociated and acutely cultured EMNs and are presently developing a whole-cell (tight-seal) voltage-clamp prep- 


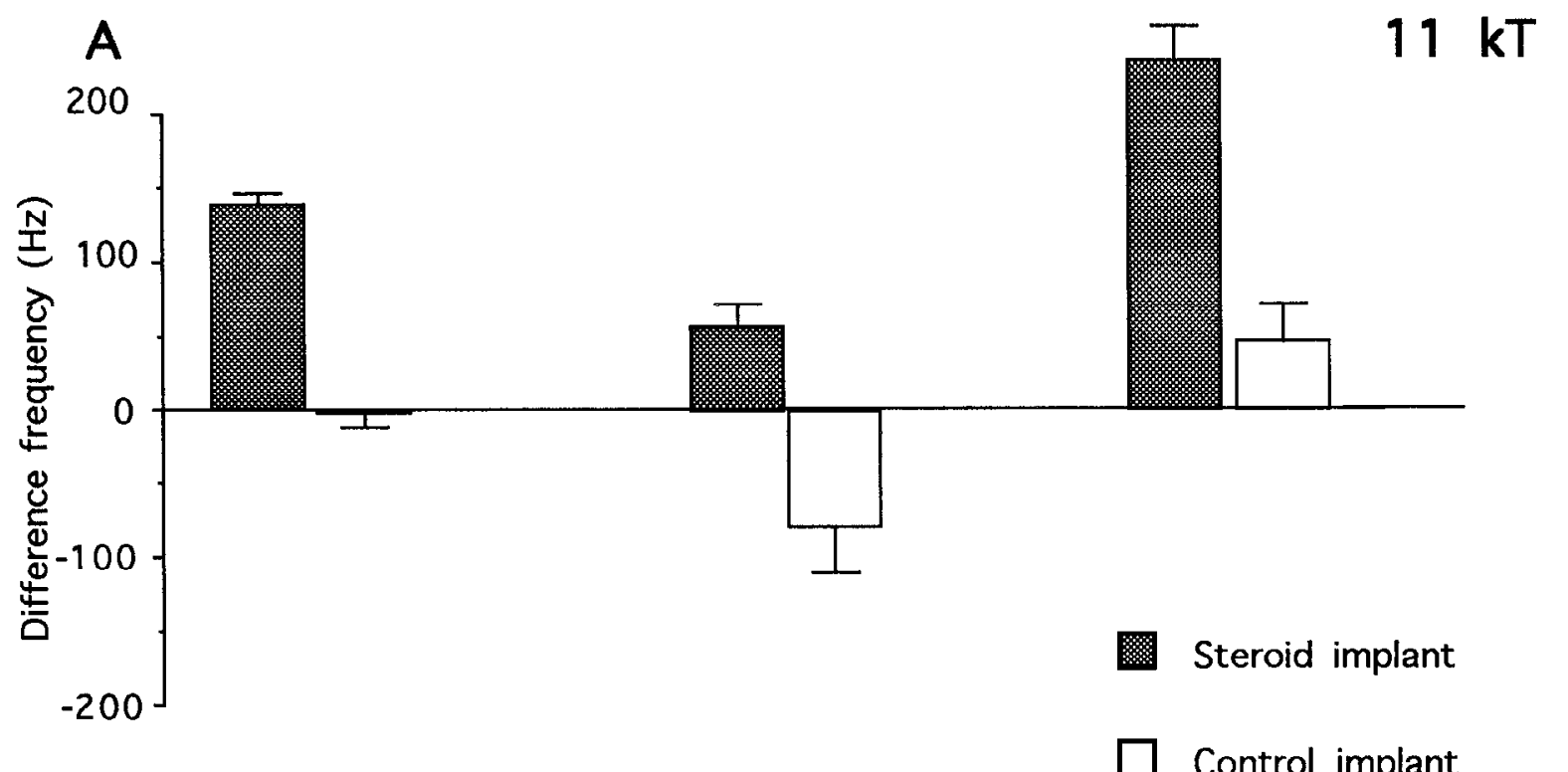

B

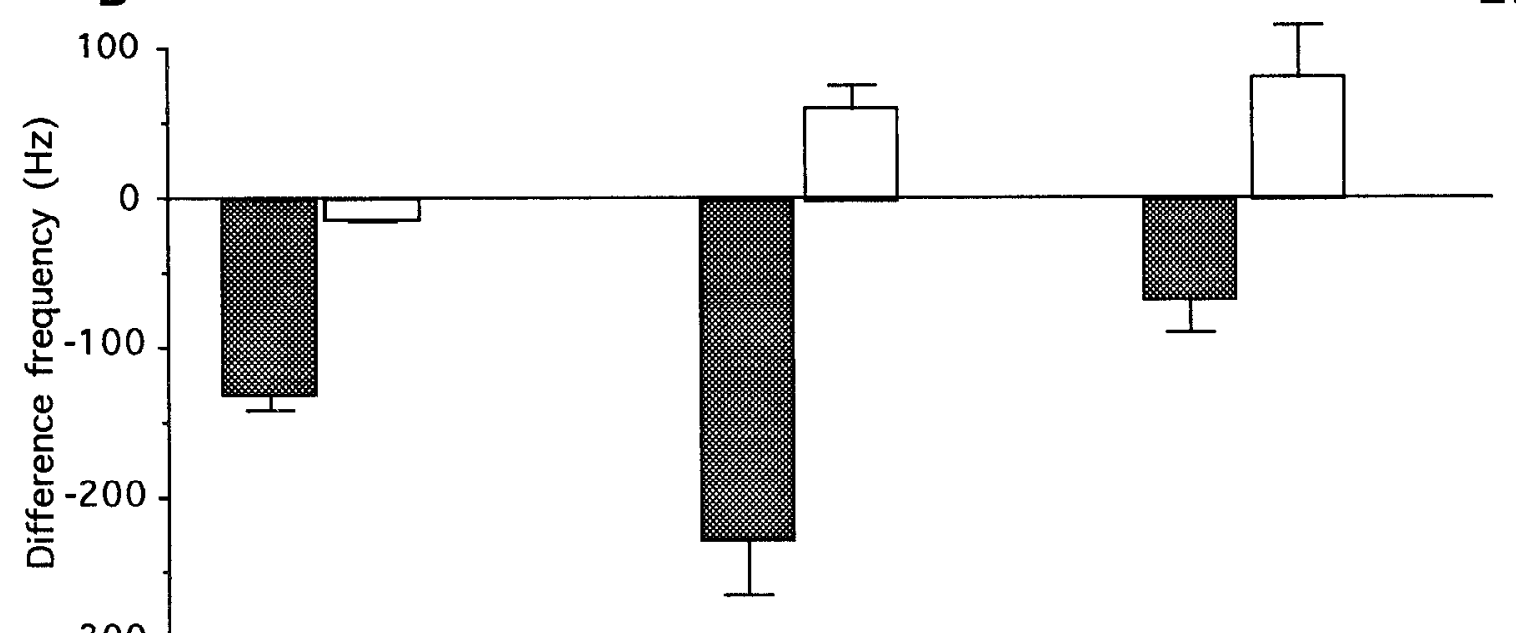

EOD

PMN

EMN

Figure 6. The effects of steroid treatment in three separate experiments looking at in vitro firing frequency of the PMN ( 3 PMNs/group), the EMNs (5 fish/group), or the intact EOD frequency ( 5 fish/group). Steroid-induced changes in frequency (difference frequency; see Materials and Methods) were compared with control values using a single-variable ANOVA. $A$, Chronic 11 ketotestosterone $(11 \mathrm{kT})$ implants significantly increased EOD frequency $(p<0.0001)$, in vitro PMN firing rate $(p<0.02)$, and in vitro EMN firing rate $(p<0.001)$. $B$, Chronic 17 - $\beta$-estradiol $(E 2)$ implants decreased EOD frequency $(p<0.0004)$, in vitro PMN firing rate $(p<0.002)$, and in vitro EMN firing rate $(p<0.0004)$.

aration for studying the ionic currents of these high-frequency, steroid-sensitive oscillators. This could elucidate interactions that underlie steroidal modulation of more complex reproductive behaviors.

\section{REFERENCES}

Alves-Gomes JA, Orti G, Haygood M, Heiligenberg W, Meyer A (1995) Phylogenetic analysis of the South American electric fish (order Gymnotiformes) and the evolution of their electrogenic system: a synthesis based on morphology, electrophysiology, and mitochondrial sequence data. Mol Biol Evol 12:298-318.

Anderson MJ, Waxman SG, Tadlock CH (1984) Cell death of asynaptic neurons in regenerating spinal cord. Dev Biol 103:443-455.
Bennett MVL (1971) Electric organs. In: Fish physiology (Hoar WS, Randall DJ, eds), pp 493-574. New York: Academic.

Bennett MVL, Pappas GD, Gimenez Nakijima Y (1967) Physiology and ultrastructure of electrotonic junctions. IV. Medullary electromotor nuclei in gymnotid fish. $\mathbf{J}$ Neurophysiol 30:236-300.

Bennett MVL, C Sandri, Akert K (1978) Neuronal gap junctions and morphologically mixed synapses in the spinal cord of a tclcost, Sternachus albifrons (Gymnotoidei). Brain Res 143:43-60.

Bennett MVL, Barrio LC, Bargiello TA, Spray DC, Hertgerg E, Saez JC (1991) Gap junctions: new tools, new answers, new questions. Neuron 6:305-320.

Bullock TH (1969) Species differences in effect of electroreceptor input on electric organ pacemakers and other aspects of behavior in electric fish. Brain Behav Evol 2:85-118. 
Bullock TH, Heiligenberg W, editors (1986) Electroreception. Wiley, New York.

Dulka JG, Maler L (1994) Testosterone modulates female chirping behavior in the weakly electric fish, Apteronotus leptorhynchus. J Comp Physiol [A] 174:331-343.

Dye J (1991) Ionic and synaptic mechanisms underlying a brainstem oscillator: an in vitro study of the pacemaker nucleus of Apteronotus. J Comp Physiol [A] 168:521-532.

Dye J, Heiligenberg W (1987) Intracellular recording in the medullary pacemaker nucleus of the weakly electric fish, Apteronotus, during modulatory behaviors. J Comp Physiol [A] 161:187-200.

Dye J, Meyer JH (1986) Control of the electric organ discharge in weakly electric fish. In: Electroreception (Bullock TH, Heiligenberg W, eds), pp 71-102. New York: Wiley.

Enger PS, Szabo T (1968) Effects of temperature on discharge rate of the electric organ of some gymnotids. Comp Biochem Physiol 27:625-627.

Ferrari MB, McAnelly ML, Zakon HH (1995) Individual variation in and androgen modulation of the sodium current in electric organ. J Neurosci 15:4023-4032.

Gage PW, Lamb GD, Wakefield BT (1989) Transient and persistent sodium currents in normal and denervated mammalian skeletal muscle. J Physiol (Lond) 418:427-439.

Hagedorn M, Heiligenberg W (1985) Court and spark: electric signals in the courtship and mating of gymnotid fish. Animal Behav 33:254-265.

Hille B (1992) Ionic channels of excitable membranes. Sunderland, MA: Sinauer.

Hopkins CD (1972) Sex differences in electric fish signaling in an electric fish. Science 176:1035-1037.

Kawasaki M, Maler L, Rose GJ, Heiligenberg W (1988) Anatomical and functional organization of the prepacemaker nucleus in gymnotiform electric fish: the accommodation of two behaviors in one nucleus. J Comp Neurol 276:113-131.

Keller CH, Zakon HH, Sanchez DY (1986) Evidence for a direct effect of androgens on electroreceptor tuning. J Comp Physiol [A] 158:301-310.
Kirschbaum K (1983) Myogenic electric organ precedes the neurogenic electric organ in apteronotid fish. Naturwissenschaften 70:205.

Maler L, Boland M, Patrick J, Ellis W (1983) Localization of zinc in the pacemaker nucleus of high frequency gymnotid fish. In: The neurobiology of zinc, Part A, Physiology, anatomy, techniques (Fredrickson CJ, ed), pp 199-212. New York: Liss.

Maler L, Jande S, Lawson EM (1984) Localization of vitamin D-dependent calcium binding protein in the electrosensory and electromotor system of high frequency gymnotid fish. Brain Res 301:166-70.

Meyer JH (1984) Steroid influences upon discharge frequencies of intact and isolated pacemakcr of weakly electric fish. J Comp Physiol [A] 154:659-668.

Meyer JH, Leong M, Keller CH (1987) Hormone induced and maturational changes in electric organ discharges and electroreceptor tuning in the weakly electric fish Apteronotus. J Comp Physiol [A] 160:385-394.

Schaefer JE, Zakon HH (1993) High frequency oscillators in the spinal cord of Apteronotus are correlated to EOD. Soc Neurosci Abstr 19:377.

Schaefer JE, Vijayvergiya C, Zakon HH (1994) Deafferentation causes axon collapse of naturally targetless electromotor neurons in the weakly electric fish Apteronotus leptorhynchus. Soc Neurosci Abstr 20:371.

Turner RW, Borg LL, Naweed SI (1995) A technique for dissociation of neurons from restricted regions of the vertebrate CNS. J Neurosci Methods 56:57-70.

Waxman SG, Pappas GD, Bennet MVL (1972) Morphological correlates of functional differentiation of nodes of Ranvier along single fibers in the neurogenic electric organ of the knife fish Sternarchus. J Cell Biol $53: 210$.

Zakon HII, Zucker MS, Blyveis N, Thomas P, Blundon J (1993) Species differences in frequency dimorphism and estrogen sensitivity of EOD in Apteronotus albifrons and A. leptorhynchus. Soc Neurosci Abstr 19:377. 